\title{
INSTITUTOS CONFUCIO EN COLOMBIA: UN ANÁLISIS DESDE LA TEORÍA DE NYE*
}

\author{
Yinny Marcela Blanco Rubiano ${ }^{(a)}$
}

CONFUCIUS INSTITUTES IN COLOMBIA: AN

ANALYSIS FROM NYE'S THEORY

INSTITUTOS CONFÚCIO EM COLÔMBIA: UMA

ANÁLISE DESDE A TEORIA DE NYE

Fecha de recepción: 5 de abril del 2019

Fecha de aprobación: 17 de junio del 2019

Disponible en línea: 1 de julio del 2019

\section{Sugerencia de citación:}

Blanco Rubiano, Y. M. (2019). Institutos Confucio en Colombia: un análisis desde la teoría de Nye. Razón Crítica, 7, 209-232, doi: 10.21789/25007807.1508

\footnotetext{
* El presente artículo se estructura a partir de la tesis titulada "Creación de institutos Confucio en Colombia y la política exterior de China. Estudio de caso: Instituto Confucio de la Universidad de Bogotá Jorge Tadeo Lozano 2012-2016” presentada para obtener el título de magíster en Relaciones Internacionales en la Universidad de Bogotá Jorge Tadeo Lozano. Esta fue dirigida por el profesor Mario Adolfo Forero Rodríguez y evaluada por las jurados Angélica Johanna Alba Cuellar y Angélica Bernal Olarte.

(a) Magíster en Relaciones Internacionales de la Universidad de Bogotá Jorge Tadeo Lozano, Colombia https://orcid.org/0000-0002-1525-0681

yinnym.blancor@utadeo.edu.co
} 


\section{R E S U M E N}

Entre las estrategias de política exterior de un Estado es posible encontrar el uso de la cultura y los valores políticos como una forma de generar atracción en otros países, mejorar las relaciones y el entendimiento y, en consecuencia, legitimar su poder y mejorar su influencia, proyección y posición internacional. En este artículo se analiza la política exterior de creación de los institutos Confucio en Colombia como parte de la estrategia diplomática de la República Popular de China para fortalecer su posición e influencia en América Latina. A través de herramientas metodológicas como encuestas, entrevistas y trabajo de campo en los institutos, se detalla el funcionamiento del Instituto Confucio de la Universidad de Bogotá Jorge Tadeo Lozano y se identifican los elementos diplomáticos presentes en su modelo operativo. Se concluye que la implementación de estos institutos en Colombia, así como en el resto del mundo, forma parte de una estrategia del país asiático para cumplir sus objetivos de su interés nacional a través de la expansión de su cultura e idioma de una forma planificada, coordinada y atrayente..

PALABRAS CLAVE: Diplomacia, poder, Joseph Nye, Instituto Confucio, Universidad de Bogotá Jorge Tadeo Lozano. 


\section{A B S T R A C T}

Among the foreign policy strategies of a State it is possible to acknowledge the use of culture and political values as a way of attracting the interest of other nations, improve relations and the understanding of other cultures, and, as a consequence, legitimize its power and strengthen its influence, projection and international position. This article studies the creation of Confucius Institutes in Colombia as part of the diplomatic strategy of the People's Republic of China foreign policy to strengthen its position and influence over Latin America. Through methodological tools such as surveys, interviews and field work in the institutes, the operation of the Confucius Institute at Jorge Tadeo Lozano University and the diplomatic elements in its operating model are explained. It is concluded that the implementation of these institutes in Colombia, as in the rest of the world, is part of a strategy of the Asian country to fulfill its national interest objectives through the expansion of its culture and language, following a structured, coordinated and attractive plan.

KEY WORDS: Diplomacy, power, Joseph Nye, Confucius Institute, Jorge Tadeo Lozano University.

\section{R E S U M O}

Entre as estratégias de política exterior de um Estado é possível evidenciar o uso da cultura e os valores políticos como uma forma de gerar atração em outros países, melhorar as relações e o entendimento. Como consequência, o Estado pode legitimar seu poder e melhorar sua influência, projeção e posição internacional. Neste artigo analisa-se a política exterior de criação dos institutos Confúcio em Colômbia como parte da estratégia diplomática da República Popular de China para fortalecer a sua posição e influência em América Latina. A través de ferramentas metodológicas como enquetes, entrevistas e trabalho de campo nos Institutos, detalha-se o funcionamento do Instituto Confúcio da Universidad Jorge Tadeo Lozano, e se identificam os elementos diplomáticos presentes em seu modelo operativo. Conclui-se que a implementação destes institutos em Colômbia, bem como no resto do mundo, forma parte de uma estratégia do país asiático para cumprir seus objetivos de interesse nacional. Isto é possível a través da expansão de sua cultura e idioma, de uma maneira planificada, coordenada e atraente.

PALAVRAS-CHAVE: Diplomacia, poder, Joseph Nye, Instituto Confúcio, Universidad Jorge Tadeo Lozano. 


\section{N T R O D U C C I Ó N}

En lo que va del siglo xxi, China se ha presentado como una potencia económica y política en el escenario mundial y ha marcado nuevas tendencias geoeconómicas y políticas. En la región latinoamericana, por ejemplo, ha habido un incremento evidente en las relaciones con el país asiático, y ha quedado claro el interés de este último por fortalecer su influencia en América Latina y establecerse como un socio importante para la región. Entre las estrategias que ha implementado el país asiático está la incorporación de las relaciones amistosas como un elemento primordial de su política exterior, a través del cual busca posicionarse como una potencia mundial, sin generar recelo o prevención en la comunidad internacional.

La creación de los institutos Confucio en América Latina y en el resto del mundo es parte importante de esta implementación de la diplomacia en la política exterior china. Estos institutos se establecen, más que como escuelas de enseñanza del idioma chino mandarín, como centros de promoción de la lengua y cultura del país oriental, de intercambio educativo y de cooperación internacional.

El despliegue de los institutos Confucio es coherente con el crecimiento económico y comercial de China, además de con su posicionamiento como potencia mundial desde inicios de este siglo. Desde el primer instituto, fundado en Seúl en el 2004, se han creado más de 500 en el mundo, y el Gobierno chino aspira llegar a los 1.000 para el 2020. En América Latina, cuyo primer instituto fue fundado en México en el 2006, se registra la creación de 159 
institutos Confucio para finales del 2016, lo que equivale al $32 \%$ de los fundados en el mundo.

En este artículo se analiza la creación y funcionamiento de los institutos Confucio, y se concluye que estos funcionan como una estrategia diplomática de la política exterior de la República Popular de China. Se sostiene, además, que su aplicación va en línea con el interés nacional chino de fortalecer su atracción en América Latina y las relaciones amistosas con los países de la región, que han crecido paralelas a los acuerdos comerciales y políticos.

Para sustentar estos planteamientos, se analizó la creación y funcionamiento de los institutos Confucio en Colombia, en especial, el creado en la Universidad de Bogotá Jorge Tadeo Lozano (Utadeo). Como herramientas metodológicas se aplicaron encuestas ${ }^{1}$ a egresados, estudiantes y profesores del Instituto Confucio de la Utadeo, con una participación del 40\%, 24\% y 100\%, respectivamente. Además se realizaron entrevistas, un trabajo de campo y una recolección de información de los institutos Confucio en Colombia. Se consultaron también fuentes secundarias y se usó como referente principal para el marco teórico la obra del politólogo norteamericano Joseph Nye.

En su obra Bound to Lead: The Changing Nature of American Power (1990), Joseph Nye planteó, en el contexto norteamericano, una diferenciación en el tipo de relaciones que un Estado establece con otros países: por un lado, el fundamentado desde la coacción y el poder tanto económico como militar, y, por el otro, el que se basa en la atracción a través de la cultura, la política exterior y los valores políticos (citado por Rodríguez \& Leiva, 2013, p. 2).

Nye señala que esta segunda forma de diplomacia se reconoce cuando un país busca generar una atracción por otros para alcanzar sus objetivos en la política exterior, sin coacción o manipulación. De esta forma, se pueden identificar este tipo de relaciones amistosas cuando un Estado busca incrementar el entendimiento mutuo, inspirar, atraer a otros y transformarlos con argumentos y contar con la capacidad de marcar preferencias asociadas a la cultura, ideología e instituciones atractivas (Nye, 2003).

1 La información completa de las encuestas aplicadas puede consultarse en el trabajo de grado "Creación de institutos Confucio en Colombia y la política exterior de China. Estudio de caso: Instituto Confucio de la Universidad de Bogotá Jorge Tadeo Lozano 2012-2016”. En este artículo, por extensión y para facilitar la lectura, solo se usaron los elementos principales identificados en las respuestas. 
A pesar de estar contextualizada desde el punto de vista occidental, esta teoría permite analizar, desde lo general hasta lo específico, la particularidad de los institutos Confucio como una estrategia de diplomacia de la República Popular de China para mejorar su proyección y posición internacional, objetivos de desarrollo de su política exterior.

Para realizar este artículo, se revisaron los conceptos esenciales de la teoría de Nye y se reconocieron sus elementos de aplicación: primero, un contexto de política exterior y de poder; segundo, la asociación con elementos culturales y el idioma; tercero, la elaboración de acuerdos de participación, y, por último, el uso de nuevas tecnologías en consistencia con un mundo globalizado. Estos elementos se analizarán e identificarán tanto en la política exterior de China como en la creación y funcionamiento de los institutos Confucio.

En primer lugar, los elementos de esta diplomacia enfocada en las relaciones amistosas deben estar enmarcados en un contexto de política exterior y de poder; este último entendido como la capacidad que tiene un país para influir sobre otros. Así pues, las estrategias de política exterior de un Estado deben estar enfocadas a generar atracción de otros países y legitimar su poder sin coacción. Si lo logra, su interacción en el sistema internacional será más dinámica y efectiva, sin resistencias para cumplir sus objetivos de interés nacional, que le representan mayor inversión de recursos.

Si un país logra legitimar su poder a ojos de los demás, hallará menos resistencia para cumplir sus deseos. Si tiene una cultura y una ideología atractivas, los demás le seguirán de buen grado. Si logra establecer normas internacionales coherentes con su sociedad, será menos probable que tenga que cambiar. Si apoya a instituciones que animen a otros países a canalizar o limitar sus actividades de forma idónea, quizá no necesite comprar tantas zanahorias y palos (Nye, 2003, p. 32).

Los elementos culturales y el idioma, si bien se consideran aspectos intangibles, no dejan de ser esenciales. Las estrategias para establecer relaciones amistosas pueden valerse de los aspectos atractivos de la cultura de un país para generar atracción en el resto del mundo. Sin embargo, dice Nye, la eficacia de este 
aspecto dependerá de la forma alineada y armónica de proyectarla y aplicarla (2003).

Es relevante también señalar que la cultura de un pueblo es "viva", es decir, constantemente se está modificando y asimilando elementos de otras culturas. De ahí que, si un Estado logra ser un promotor activo, socializador, y presenta facilidades y beneficios a los individuos del mundo para explorar de forma vivencial su cultura, será mucho más competitivo y atrayente. La razón de esto es que el impacto es más fuerte para un individuo si viene de su propia experiencia; además, las impresiones que puede dar a los demás sobre lo que vivió y aprendió serán más profundas que conocer la cultura de un país solamente a través de la información teórica que se le presente.

En la misma línea de los elementos culturales, se encuentran los valores políticos que, como se verá con más detalle al revisar, en el caso chino son la base del planteamiento teórico de su política exterior. Los valores que un Estado defiende en un contexto nacional, en la política exterior y frente a instituciones internacionales también afectan las preferencias de otros, y pueden generar atracción o repeler a terceros (Nye, 2003, p. 33).

Ahora bien, frente a los elementos enmarcados en la elaboración de acuerdos y participación, Nye (2003) argumenta que el liderazgo eficaz de un Estado se asocia con hablar, escuchar y respetar firmemente tratados internacionales, normas y foros de negociación. Para ser respetado, admirado y, por ende, generar atracción en terceros, un país debe realizar compromisos en el marco de la cooperación internacional, participar activamente y, sobre todo, respetar esos compromisos adquiridos.

Finalmente, al hacer referencia a los aspectos de uso de nuevas tecnologías en un mundo globalizado se debe tener en cuenta que la revolución de la información global e Internet han transformado la política mundial, por lo que esta debe adaptar sus estrategias alineadamente. Internet, por ejemplo, al ser un medio que maneja el poder de la atracción, es cada vez menos tangible y coercitivo; más bien, tiene una alta capacidad de influenciar y transformar al mundo. Así, si los Estados ofrecen información parcial, selectiva o que no es transparente a través de estos medios, perderán credibilidad; el “[...] Internet crea un sistema en el que el poder sobre la información se distribuye muchísimo más" (Nye, 2003, p. 14).

En definitiva cuando un país se relaciona con otros sobre la base de la amistad y la atracción a través de la cultura y los valores, puede definirse 
como una estrategia de poder engranada con la política exterior de un país, que tiene como objetivo legitimar, generar atracción y aumentar la influencia a través de la promoción de sus valores políticos, instituciones atractivas y su cultura e idioma.

A partir de estas definiciones de diplomacia y la identificación de sus elementos de aplicación, se realizará el análisis de la creación y modelo operativo de los institutos Confucio, particularmente el de la Utadeo, como parte de la estrategia diplomática de política exterior de la República Popular de China y su interés en fortalecer su influencia en América Latina, específicamente en Colombia, el cual es un país importante y geoestratégico de la región.

Lo que sigue del artículo está estructurado en dos partes: en primer lugar, se analiza la política exterior de china, sus relaciones amistosas y su acercamiento a América Latina en los inicios del siglo Xxi. En segundo lugar, se analiza la creación de los institutos Confucio en Colombia y el modelo operativo del Instituto Confucio de la Universidad de Bogotá Jorge Tadeo Lozano, y se identifican, en estos procesos, los elementos teóricos ya mencionados. Se sostiene, finalmente, que la creación de institutos Confucio en Colombia y la política exterior China es la materialización de la estrategia diplomática para cumplir sus objetivos de interés nacional chino y mejorar tanto su proyección como posición internacional.

\section{Diplomacia en la política exterior de China y su aplicación en América Latina}

La aparición de la teoría de Joseph Nye en la política exterior de China se dio en 1992, con la primera traducción de su obra al chino mandarín. Posteriormente, en 1993, Wang Huning, asesor del entonces presidente Jiang Zemin, publicó un artículo en el que invitaba a fortalecer la estrategia de poder planteada por Nye y agregaba, además, "[...] que la cultura china debería ser su principal recurso" (Rodríguez \& Leiva, 2013, p. 3). También en 1997 y 1999 se publicaron otros textos en los que se detalla la teoría y se hace un llamado a usarla en la política exterior del país.

Es necesario destacar que, si bien la República Popular de China existe desde 1949, solo a partir de los años 70 y 80 puede hablarse de una apertura internacional; las primeras dos décadas 
sostuvo una posición de aislamiento internacional, en la que hubo un refuerzo del radicalismo maoísta y una estimulación de la Revolución Cultural (Mesa \& González, 2016, p. 546).

En 1971, la Asamblea General de las Naciones Unidas reconoció la República Popular de China como el único representante legítimo ante su Consejo de Seguridad y le asignó un puesto permanente. Este reconocimiento le abrió un camino al país para participar en diferentes organizaciones internacionales, establecer relaciones diplomáticas con otros estados y acercarse a Estados Unidos.

En 1976, con la muerte de Mao Zedong y el ascenso del presidente Deng Xiaoping, la apertura internacional se intensificó. Se dio un proceso de descentralización administrativa y el país se posicionó hacia 1978, como un actor internacional con mayor actividad en lo militar, comercial, político y cultural. Esta tendencia se mantuvo en la década de los 80, con el surgimiento de alianzas estratégicas con otros países y el fortalecimiento de su participación en la economía mundial (Mesa \& González, 2016).

Así, en la década de los años 90 y principios del nuevo siglo, China ya se había comenzado a posicionar como una potencia económica "[...] con tasas de crecimiento económico por sobre el 8\% sostenidas por más de 20 años" (Rodríguez \& Leiva, 2013, p. 4), y política, con la participación en organismos multilaterales como la Organización de las Naciones Unidas (ONU), la Organización Mundial del Comercio (omc) (p. 3). No es gratuito, entonces, que haya surgido, internamente, una preocupación por la imagen que el país quería proyectar en el resto del mundo, y el tema de la diplomacia en la política exterior se haya puesto sobre la mesa.

Ya a partir del 2004, con el inicio del gobierno de Hu Jintao, China se plantea una estrategia definida en política exterior y se incorpora en su discurso, con mayor intensidad, la idea de la atracción a través de la cultura, los valores políticos y las relaciones amistosas. Principalmente, el Gobierno busca contrarrestar la referencia que se estaba haciendo del país, la "amenaza china" y reemplazarla con el concepto de ascenso pacífico, luego modificado a desarrollo pacífico.

Jintao concebía que, para lograr un desarrollo económico perdurable en el tiempo, era necesario priorizar la conformación de un ambiente de relaciones internacionales donde el mundo viera a China como un Estado pacífico y no como una amenaza (Mesa \& González, 2016, p. 558). Este concepto de desarrollo pacífico se explica en el libro White Paper: China's Peaceful Development Road, publicado en el 2005: 


\begin{abstract}
Primero, el desarrollo pacífico es un camino que China tomará inevitablemente en su proceso de modernización; segundo, China logra este desarrollo creando un ambiente global pacífico y facilitando la paz mundial a través del mismo; tercero, China logra su desarrollo confiando en sus propias capacidades, como en la reforma e innovación; cuarto, China se acomoda a las tendencias de la globalización y se esfuerza por lograr beneficios mutuos y desarrollo común con otros países; quinto, China adhiere a los principios de paz, desarrollo y cooperación, y lucha por la construcción de un mundo armonioso sustentado en la paz y prosperidad común (citado por Rodríguez \& Leiva, 2013, p. 5).
\end{abstract}

Al lado del desarrollo pacífico, otro concepto clave es el de mundo armonioso, soportado por el respeto a la soberanía territorial, no agresión y no intervención (Mesa \& González, 2016, p. 548). El concepto, que pasa de ser parte de las bases sociopolíticas internas de China a extenderse a la política externa y las relaciones internacionales, está fundamentado en la filosofía de Confucio (555 a. C.-479 a. C.) y hace referencia a que, a pesar de que el mundo esté lleno de contradicciones y diferencias, hay que equilibrarlas para lograr la armonía. Además, establece como bases sociales el respeto por la familia como núcleo de la sociedad, los valores, la unidad, la paz y el orden (Rodríguez \& Leiva, 2013).

Además en la China antigua no es posible identificar pensadores que hicieran referencia a conceptos similares al de identificar en la China antigua pensadores que hacían referencia a conceptos similares al de diplomacia (Gómez, 2013, p. 50). El mismo Confucio escribió, por ejemplo, "[g]obernar con virtud es como la estrella polar en su sitio, alrededor de la cual giran todas las demás" (Gómez, 2013, p. 51).

Queda en evidencia, entonces, que a partir del 2004 China ha demostrado una preocupación por la imagen que proyecta en el escenario internacional y ha diseñado estrategias diplomáticas que incluyen el desarrollo de las relaciones amistosas para aumentar su legitimidad, aceptación e influencia en la comunidad internacional, y generar atracción en otros países. 
El aspecto cultural, señalado como uno de los elementos principales en la teoría de Nye, es el recurso principal en esta estrategia de política exterior de china, sobre todo por la fascinación e interés que su cultura milenaria ejerce en el resto del mundo. La riqueza de su lenguaje y cultura, mantenidas a lo largo de dos milenios, fortalecen su imagen exterior $y$, por ende, aumentan su poder en el cumplimiento de sus intereses y necesidades (Ramírez, 2016).

En consecuencia, el Gobierno chino ha enfocado su diplomacia pública para "[...] informar o influir en la opinión pública de otros países, teniendo como principales instrumentos las publicaciones, películas, intercambios culturales, radio, televisión, turismo y deporte, además de la promoción del idioma y la celebración de conferencias, simposios y talleres en el extranjero" (Rodríguez \& Leiva, 2013, p. 4).

En este punto, los institutos Confucio - y su equivalente para la educación media, las aulas Confucio- adquieren una importancia enorme. Son escuelas establecidas por el Gobierno chino en cooperación con universidades extranjeras e instituciones educativas, cuyo objetivo es promover la comprensión de la lengua y cultura china. Además, tienen el compromiso de fortalecer la cooperación entre China y los países del mundo, en medio del intercambio educativo y cultural para la construcción de un mundo armonioso.

No es de extrañar, pues, que la creación de institutos Confucio esté alineada con los intereses geopolíticos de la República Popular de China o, en otras palabras, que las estrategias en política exterior en los ámbitos económico y político vayan acompañadas de una promoción de la cultura china a través del establecimiento de estos institutos.

Para ilustrar esta estrategia conjunta tenemos el caso de América Latina: el incremento de los acuerdos políticos y económicos, y la intención de China por posicionarse como socio estratégico en la región han estado acompañados de una campaña de creación de institutos y aulas Confucio en diferentes países latinoamericanos desde el 2006.

Justamente en ese año, China consolidó sus primeras negociaciones de tratados de libre comercio en la región, en Chile y México (justamente los países donde se crearon los primeros institutos Confucio), y, en el 2008, publicó el primer documento en el que establecía de forma oficial su política para las relaciones con América Latina, Texto integro del documento sobre la política de china hacia américa latina y el caribe, citado por Xu (2013), señala: 
Enfocando las relaciones con América Latina y el Caribe desde una altura estratégica, el Gobierno chino se esforzará por establecer y desarrollar con los países latinoamericanos y caribeños la asociación de cooperación integral caracterizada por la igualdad, el beneficio recíproco y el desarrollo compartido (p. 139).

En los años posteriores, China participó en eventos internacionales importantes, como el Foro Económico Mundial sobre América Latina y la Conferencia Anual sobre Riesgo País de Coface. Luego, en el 2012, el Banco Interamericano de Desarrollo (BID) y el Banco del Pueblo de China (Banco Central de China) presentaron un fondo común para Latinoamérica y el Caribe. Además, China ha mantenido relaciones permanentes con el Mercosur, el Grupo de Río, la Comunidad Andina de Naciones (CAN) y la Comunidad del Caribe (Caricom) (Bogado, 2014).

Las cifras son aún más claras a la hora de mostrar la importancia de las estrategias con los países de la región. Chile fue el primer país en firmar en el 2005, un tratado de libre comercio con el gigante asiático; en el 2006, el 36\% de exportaciones estaban orientadas a Asia, y, para el 2013, China ya era el mercado más importante para sus exportaciones, así como también de Brasil, Argentina, Costa Rica, Cuba y Perú (Hernández, 2013, p. 131). En Colombia, el país asiático es desde el 2010, uno de los socios comerciales más importantes, seguido de Estados Unidos.

El crecimiento del volumen del comercio entre la región latinoamericana y China es impresionante: en el 2005, el total de importaciones y exportaciones era de 50.466 millones de dólares, y, en solo seis años, es decir, en el 2011, había llegado a 241.485 millones de dólares, cifra que ubicó a China como el segundo mayor socio comercial de América Latina (Xu, 2013, p. 144).

Por su parte, y en este mismo periodo, la creación de institutos Confucio en América Latina ha crecido con la misma dinámica. Para diciembre de 2016 se habían creado 159 institutos Confucio en la región, el equivalente al 32\% de los fundados en el mundo, y 378 aulas Confucio, equivalentes al $58 \%$ de las establecidas en el mundo. Así, pues, los institutos Confucio hacen presencia en Argentina, Bahamas, Barbados, Bolivia, Brasil, Canadá, Chile, Colombia, Costa 
Rica, Cuba, Ecuador, Estados Unidos, Granada, Guayana, Jamaica, México, Panamá, Perú, Surinam, Trinidad y Tobago, Uruguay y Venezuela.

Es posible, entonces, identificar una equivalencia entre la creación de los institutos Confucio con los elementos de aplicación de las estrategias de relaciones amistosas de la política exterior china. El país asiático se ha interesado en presentarse como un socio estratégico y confiable para América Latina, en armonía con sus nociones de mundo armonioso, beneficio mutuo, desarrollo pacífico, amistad, cooperación y demás principios que concretan su interés nacional.

\section{Creación de los institutos Confucio en Colombia como una estrategia de política exterior de China}

Los institutos Confucio se definen como organizaciones educativas de carácter no lucrativo y pertenecen a la Oficina Nacional de Promoción Internacional de la Lengua de China (Hanban, por sus siglas en chino). Esta institución, de carácter gubernamental, tiene la función de establecer institutos Confucio en el mundo desde el 2004 y es la entidad propietaria del nombre, logotipo y marca de los institutos Confucio (Hanban, 2016).

Este organismo se encarga de suministrar los servicios necesarios para la enseñanza del idioma mandarín y la cultura china, formar a profesores de China, proporcionar los recursos didácticos a todos los países interesados en aprender el idioma chino como parte de los intercambios y de la cooperación, promover la comprensión de la cultura china a través de programas de aprendizaje, desarrollar actividades de intercambio cultural, fomentar las relaciones amistosas entre China y países que hacen parte de la cooperación y contribuir a la construcción de un mundo armonioso.

La recopilación de estas funciones y objetivos muestra la articulación de Hanban y la política exterior del Gobierno chino con el contexto teórico y los elementos de la diplomacia y la atracción a través de la cultura y los valores. De hecho, este proyecto ha sido calificado como "[...] uno de los programas de penetración cultural más ambiciosos que se haya llevado a cabo jamás” (Ibáñez \& Dávila, 2010, p. 36).

En Colombia, actualmente existen tres institutos Confucio: el Instituto Confucio de la Universidad de los Andes, el Instituto Confucio de Medellín y el Instituto Confucio de la Utadeo.

En el proceso de creación de cada instituto participan, además de Hanban, las embajadas de China en Colombia y de Colombia en China, 
una universidad del país asiático y su par colombiana. Aquí, cabe recordar que los institutos Confucio no se crean de forma independiente, sino que se asocian con universidades destacadas de cada ciudad (en Bogotá con la Universidad de los Andes y la Utadeo, y en Medellín con la Universidad de Antioquia y la Universidad Eafit).

Esta característica de los institutos, que podría pasar como una decisión simplemente pragmática, debe leerse también como parte de la estrategia de Hanban para alcanzar sus objetivos en el marco de la política exterior china: aprovecha la estabilidad de estas universidades y se beneficia de su reconocimiento, trayectoria, instalaciones, ubicación, población objetivo, apoyo cultural, apoyo en disposición de recursos, entre otros.

También vale la pena destacar la presencia de personalidades importantes de los gobiernos implicados en los procesos de creación de los institutos, ya que esto fortalece su carácter diplomático y genera más impacto en la comunidad y proyección de los servicios que ofrecen. Por ejemplo, el Instituto Confucio de Medellín se creó en el 2010 en el marco del acuerdo de cooperación realizado durante la visita a Colombia del vicepresidente chino, Xi Jinping, y, así mismo, en el evento de inauguración del Instituto de la Universidad de Bogotá Jorge Tadeo Lozano, “[...] estuvieron presentes la Ministra de Relaciones Exteriores de Colombia, Dra. María Ángela Holguín, y el miembro del Buró Político del Partido Comunista de China, Sr. Li Changchun" (Instituto Confucio Utadeo, 2013).

Ahora bien, en lo referente al funcionamiento, los tres institutos ${ }^{2}$ presentes en Colombia tienen características similares, alineadas a los principios y funciones de Hanban. Hay dos directores, uno por cada país, y un personal administrativo, casi en su totalidad colombiano. Los profesores, por su parte, son siempre de nacionalidad china, usualmente mujeres, y han recibido formación tanto en español como en la enseñanza del mandarín.

2 En el trabajo de grado, ya mencionado y a partir del cual se estructura este artículo, se señala con mayor detenimiento el funcionamiento de cada uno de los institutos Confucio. Para ampliar esta información, así como el análisis del modelo operativo identificado en el Instituto Confucio de la Utadeo, se invita al lector a consultarlo. 
Los servicios ofrecidos también son en esencia los mismos: cursos de chino mandarín para adultos; realización de los exámenes Hsk y Hsk K, pruebas oficiales para evaluar la competencia de la lengua china requerida en universidades; asignación de becas para estudiar en China a estudiantes sobresalientes, y los campamentos de verano, realizados una vez al año en la universidad china asociada a cada instituto.

A continuación, se profundiza en algunas de estas características y servicios de los institutos, y en su relación con los elementos de aplicación de la diplomacia como política exterior, desde el análisis del modelo operativo del Instituto Confucio de la Universidad de Bogotá Jorge Tadeo Lozano (figura 1). Se representan de forma gráfica los procesos que intervienen para el desarrollo de los institutos Confucio y, en el análisis, se resaltan los componentes que representan los elementos ya identificados en la teoría de Nye.

Como se puede observar en la imagen, el modelo operativo del Instituto Confucio de la Universidad de Bogotá Jorge Tadeo Lozano comienza en un contexto de política exterior, propiciado por el Ministerio de Educación de la República Popular de China y bajo los lineamientos definidos para la creación de los institutos Confucio en el mundo como una organización educativa no lucrativa, perteneciente a Hanban, y con la participación de la Embajada popular de China y la Embajada de Colombia.

Esto significa, en otras palabras, que la coordinación y control en el cumplimiento de los objetivos establecidos en la fundación de los institutos Confucio son de interés nacional para la República China en el desarrollo de sus estrategias de política exterior.

Luego se encuentra la planeación administrativa, en la que participa personal administrativo de Colombia y de China, y que se enfoca a la renovación y elaboración de acuerdos, recursos, comunicación, ejecución y eventos entre Colombia y China. Para la creación de cada instituto es necesaria, además del establecimiento y respeto de los acuerdos iniciales entre las entidades participantes, una continua cooperación bilateral entre las direcciones de China y Colombia para gestionar la parte administrativa, llevar un control del presupuesto y recursos, además de manejar la comunicación, organización de eventos y estrategias de mercadeo para sumar estudiantes matriculados.

También en este punto se evidencia que el Instituto Confucio de la Utadeo desarrolla procesos de compras, contratación y de comunicación, mercadeo y promoción, enfocados en el uso de nuevas tecnologías. Se utilizan 


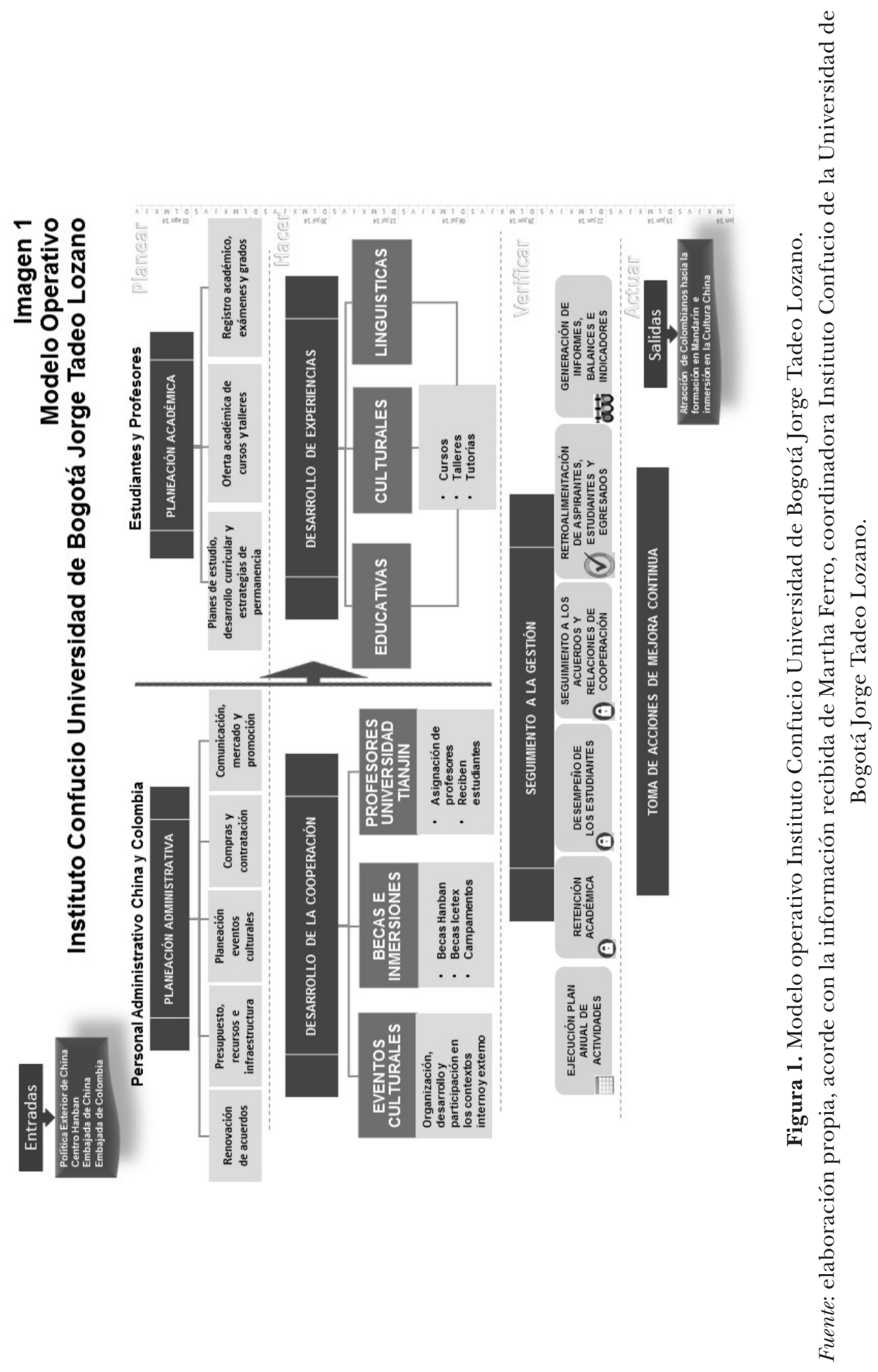


correos electrónicos y redes sociales como Facebook, Twitter, Instagram y la página web del Instituto; además, hay contacto directo con la población objetivo a través de telemercadeo y los demás medios de comunicación de la Utadeo.

Una vez son ejecutados estos procedimientos, se pasa a la etapa de desarrollo de la cooperación. Este punto es esencial, ya que se enfoca en promover los eventos culturales e intercambios académicos, claves en la utilización de los elementos culturales y el idioma en la estrategia de aplicación de la diplomacia como parte de la política exterior china.

En primer lugar, está la realización de eventos culturales. Se dan en un ámbito interno y en uno externo, y se destacan por enfocarse en temas relacionados con China que usualmente generan gran interés. Se destacan, entre otros, presentaciones teatrales y musicales, lecturas de poesía, proyección de películas, conferencias sobre ideología, historia y religión, muestras de culinaria china y artes marciales, además de actividades académicas como clubes de conversación. Estas actividades, en su contexto externo, suelen ser abiertas a un público más amplio (la comunidad universitaria, por ejemplo), por lo que sirven también para atraer nuevos miembros al Instituto, y generar fascinación y atracción por el país asiático en Colombia.

También se destacan, como actividades internas, la realización de competencias, como la del Puente Chino. Este concurso tiene como propósito proporcionar a los estudiantes la oportunidad de demostrar su dominio del idioma y el conocimiento sobre China y su cultura. Inicialmente se desarrolla un concurso interno, el ganador participa en el concurso con los demás institutos Confucio del país y el ganador viaja a China a participar en el concurso mundial en representación del país.

Con respecto a los intercambios académicos, hay que destacar la Beca Hanban y el campamento de verano. La primera es una convocatoria mundial para el aprendizaje y enseñanza del chino con duración de seis meses, un año o dos años (maestría). Cubre los gastos de matrícula del curso, alojamiento, manutención de 1.135.000 COP (2.500 CNY) mensuales y, en algunos casos, los tiquetes aéreos.

El Instituto Confucio de la Utadeo es el encargado de evaluar y recomendar al estudiante que se postula a esta beca. Es fundamental que tenga planes que incluyan a China, por ejemplo, continuar sus estudios en el país o desempeñarse en funciones como traducción, en comercio exterior, negocios, carrera diplomática, entre otros. 
Por su parte, los campamentos de verano en China tienen un impacto mucho más amplio; se realizan anualmente, en convenio con la universidad china participante en el Instituto, y están dirigidos a 20 estudiantes de cualquier nivel de chino (con una evaluación previa por parte del Instituto). El campamento es subsidiado por Hanban y cubre alojamiento, alimentación, actividades, curso, transporte interno y la mitad del tiquete. Se estima que, del valor total del campamento (aproximadamente 13.000.000 COP), el estudiante cubre solo el 30\%.

La realización de estos intercambios académicos funciona como un elemento de atracción excepcional, ya que se sustenta en la propia experiencia e inmersión en la cultura china y deja impactos más profundos en la persona que la vive. Además, después de que el estudiante participa en el campamento, debe compartir su percepción y las experiencias vividas a través de artículos, videos y fotografías en diferentes medios de comunicación del Instituto Confucio. Esta difusión de las experiencias es fundamental; motiva a más personas a aprender el idioma y la cultura china, además de que atrae a otros para aprovechar esta oportunidad.

Finalmente, no se debe pasar por alto el hecho de que Hanban apoye económicamente los intercambios y promueva los viajes de estudio e inmersión de estudiantes colombianos a China. Este aspecto evidencia el carácter no lucrativo de la institución y su interés por promover el idioma, la cultura y los valores chinos, lo que puede leerse como una aplicación de la diplomacia, en la medida en que genera interés y atracción en las personas.

Para retomar el análisis del modelo operativo del Instituto Confucio, se identifica ahora la etapa de la planeación académica. En esta se encuentra el desarrollo de experiencias educativas, culturales y lingüísticas, en las que se demuestra la aplicación de los elementos de diplomacia mediante la ejecución de cursos, talleres, tutorías.

En el Instituto Confucio de la Utadeo, se ofrecen cursos de chino mandarín para adultos, jóvenes y niños. Hay tres modalidades: cursos intensivos, con una intensidad de 10 horas semanales; cursos regulares, con unas cuatro horas semanales, y cursos con créditos académicos, para estudiantes de la Universidad Tadeo Lozano, también con una intensidad de cuatro horas semanales. 
En los cursos se utilizan libros de texto y de ejercicios prácticos como guías para el desarrollo de cada clase. Adicionalmente, cada clase tiene su propia planeación de contenidos documentados a través del syllabus del curso, donde se describen los objetivos de la clase, la fecha, el tema, la página del libro guía, el contenido (vocabulario básico, vocabulario adicional y gramática), las habilidades comunicativas (hablar, escuchar, leer y escribir), los temas culturales, la metodología pedagógica que se va a utilizar (apoyo videos, fotografías, otros libros, dinámicas, ejercicios prácticos), la página del libro guía para tarea y los exámenes de evaluación del nivel.

Al hacer la revisión de estos syllabi y recopilar la información correspondiente del 2016, se observa que las clases de mandarín tienen un enfoque interdisciplinar, con énfasis en la cultura china. Se encuentran temas de religión, medicina, cuidado del medio ambiente, geografía, música, danza, pintura, deportes, negocios, costumbres, entre muchos otros. Esto quiere decir que los programas curriculares están pensados para que el estudiante no solo aprenda vocabulario, gramática y pronunciación, sino que se vea inmerso en la cultura e historia del país, en sus tradiciones y costumbres, e incluso en los cambios que China ha experimentado en los últimos años. Los temas sobre Colombia son menos abordados y, casi siempre, solo tratados en relación con los asiáticos.

En las encuestas realizadas para el desarrollo de esta investigación, los estudiantes reconocen que sus conocimientos sobre China antes de asistir al Instituto estaban en niveles medios y bajos, mientras que su valoración actual es de niveles medio, alto y muy alto en los temas relacionados con idioma, cultura, religión, geografía, costumbres, oportunidades de educación, oportunidades laborales oportunidades de comercialización.

En la última etapa del modelo identificado en el Instituto, se encuentra la etapa de seguimiento a la gestión que, en términos generales, es el monitoreo de la capacidad de atraer e influir sobre otros, evidenciada en la cobertura, proyección y análisis de la percepción de experiencias e impacto de la comunidad educativa.

Frente a la cobertura del Instituto Confucio de la Utadeo, es posible identificar un crecimiento desde su fundación, en el 2012. En el 2013 se registraron 72 estudiantes matriculados; en el 2014, 72 estudiantes; en el 2015, 223 estudiantes, y en el 2016, 600 estudiantes hacían parte de su comunidad educativa.

Se ve, en los dos últimos años, que la estrategia implementada por el Instituto, de vincular dentro de los planes de estudios la opción de 
un segundo idioma en los diferentes programas académicos de la Utadeo, ha generado un mayor número de personas matriculadas. Sea porque el estudiante tenga interés de aprender y conocer la cultura, o sencillamente por cumplir los requisitos de créditos académicos exigidos en su programa académico de pregrado, la estrategia ha permitido que más personas conozcan y vivan de cerca la experiencia de estudiar en el Instituto Confucio.

Igualmente, ya en el tema de la percepción, las encuestas aplicadas mostraron que el $95 \%$ de los estudiantes y egresados están satisfechos con los servicios recibidos en el Instituto Confucio Utadeo, y el 97,3\% considera que los conocimientos adquiridos resultan extremadamente útiles para su proyecto de vida por las oportunidades personales y de negocios que pueden obtener con el país asiático.

Asimismo, entre las ventajas de estudiar en el Instituto Confucio se señalan, principalmente y además del aprendizaje del idioma, las ventajas laborales, el conocimiento sobre cultura y costumbres, la posibilidad de conocer el país y el enriquecimiento de su visión de mundo.

Finalmente, como salida del modelo operativo expuesto del Instituto Confucio de la Utadeo, se identifica explícitamente la atracción de colombianos hacia la formación en mandarín e inmersión de la cultura china.

Vale la pena retomar, en este punto, los aspectos de la teoría de Nye identificados en el modelo operativo del Instituto Confucio: los elementos culturales y el idioma, en la enseñanza del chino mandarín, el enfoque cultural de las actividades y cursos de los institutos y las experiencias de inmersión de los estudiantes en China a través de intercambios y becas; el uso de nuevas tecnologías en consistencia con un mundo globalizado, en el uso de redes sociales y plataformas de información virtuales en los Institutos, y la elaboración de acuerdos de participación, en el establecimiento y respeto de acuerdos, constitución, recursos, ejecución y eventos entre Colombia y China en la creación y funcionamiento de los institutos Confucio.

Puede sostenerse, entonces, a partir de este modelo, que en los institutos Confucio hay una profundización en los contenidos pedagógicos en temas que generan atracción en el país asiático, y se logra generar un interés por descubrir e integrarse en la inmersión y relación con China. Esto, sumado al manejo de diferentes medios 
de comunicación, y divulgación y a los incentivos, beneficios y ayudas económicas para participar en el programa completo de servicios que ofrece el Instituto Confucio en la Utadeo, representa una evidencia objetiva y tangible de que los institutos Confucio son parte esencial de una estrategia de política exterior, basada en las relaciones amistosas y la atracción cultural, de la República Popular de China.

Es importante, además, tener en cuenta que este modelo de operación se replica en los demás institutos Confucio alrededor del mundo, ya que, en todos, Hanban sigue los mismos lineamientos de creación y funcionamiento, a través de convenios con reconocidas universidades de cada país y con autonomía frente a los contenidos abordados en las clases y actividades extracurriculares.

Es en este punto que los institutos Confucio pueden verse más claramente como parte de una estrategia global del Gobierno chino para fortalecer su influencia en las regiones en las que tiene interés político o comercial. Al promocionar y difundir su cultura, costumbres e idioma, la atracción de las personas hacia el país aumenta significativamente, lo que le genera grandes ventajas a la hora de hacer negocios o establecer relaciones.

El elemento cultural, esencial en la formación de los estudiantes de los institutos, es uno de los grandes generadores de esa atracción, es decir, el hecho de que la enseñanza del idioma esté acompañada de conocimientos de la cultura del país es atractivo para los estudiantes, que consideran este aspecto como una de las grandes ventajas de formar parte de los Institutos.

Igualmente, esa inmersión cultural está acompañada de ventajas y beneficios para conocer el país. El Gobierno chino, en la figura de Hanban, está interesado en que los estudiantes, ya con cierto grado de atracción por el país, viajen a China, conozcan de primera mano su cultura y costumbres y practiquen el idioma. El apoyo económico que se ofrece para esto es más que significativo, por lo que los estudiantes tienen grandes posibilidades de ir.

Una de las consecuencias de estos intercambios es el aumento de esa atracción; cada uno de los jóvenes que participa de los campamentos, becas o inmersiones replica y difunde su experiencia, además de que promociona la riqueza tanto natural como cultural de China. Adicionalmente, la mayoría de ellos se prepara para un proyecto de vida relacionado con el país, sea como lugar de estudio, de trabajo o para realizar negocios. 
Vale la pena analizar, finalmente, las características de la comunidad estudiantil de los institutos Confucio: joven y productiva, sin hijos y, en su mayoría, de estratos socioeconómicos altos. No queda duda, entonces, de que entre los intereses de China, al fundar los institutos Confucio en Colombia y en el mundo, está lograr la atracción de un sector de la población que puede generar, a futuro, relaciones comerciales, políticas y culturales con los demás países, relaciones que con certeza fortalecerán su posición como potencia económica y política en el escenario global.

\section{Conclusiones}

En el contexto de las relaciones internacionales es posible identificar diferentes formas en las que un Estado interactúa con otros países y con la comunidad internacional en general: una es a partir de la coacción y el poder militar; otra, a través de la diplomacia y las relaciones amistosas con sus pares. Esta segunda estrategia, cuyo planteamiento teórico fue realizado por Joseph Nye en 1990, está basada en la generación de atracción por medio de la cultura y los valores políticos, y el incremento del entendimiento entre países.

En este artículo se identificó el uso de esta segunda estrategia en la política exterior de la República Popular de China. El país asiático ha sabido explotar sus riquezas culturales para atraer al mundo y posicionarse como una potencia y, a través de un programa planeado y ejecutado desde el propio Gobierno, los institutos Confucio han sido su herramienta principal.

Se observó que, a partir de los conceptos de desarrollo pacífico y mundo armonioso, la política exterior china ha logrado contrarrestar los temores que en algún momento hubo en Occidente frente a su crecimiento económico y acercar su cultura al resto de países. En América Latina, por ejemplo, el incremento de las relaciones políticas y comerciales ha sido significativo desde inicios de este siglo y, actualmente, China es uno de los principales socios de la región.

Los institutos Confucio se identificaron como elementos clave en el fortalecimiento y mantenimiento de estas relaciones, ya que generan atracción y admiración hacia la cultura china. Su creación y funcionamiento forman parte de la estrategia diplomática de 
la República Popular de China para dar cumplimiento a sus objetivos de interés nacional, enfocados a mejorar su influencia en la región y proyección y posición internacional.

La aplicación de esta política exterior le ha permitido al país asiático promocionar su idioma, cultura y valores de forma eficaz, promover una imagen atractiva en el resto del mundo y, claro, legitimar su poder económico y político en aumento.

Es claro que estrategias de este tipo, cuando se implementan de forma alineada con una política exterior clara y se cuenta con recursos para promocionarla en el mundo, puede traer grandes beneficios a los países que deciden invertir en ellas. El mundo del siglo xxı, globalizado y permanentemente comunicado, realza cada vez más la importancia de la diplomacia como camino para alcanzar los objetivos e intereses de las naciones.

El funcionamiento de este Instituto Confucio puede ser un ejemplo de cooperación internacional y de diplomacia a través de la educación y la cultura. Resulta interesante generar más análisis para encontrar estrategias de política exterior que, sin ser coercitivas ni violentas, mejoren la posición de los países en el ámbito internacional y les permitan cumplir con mayor eficacia sus objetivos.

\section{Referencias}

Bogado B. L. (2014). Las relaciones entre China y América Latina y los enigmas de los lazos históricos. Buenos Aires: Ediciones Iri. Recuperado de http://www.iri. edu.ar/images/Documentos/publicaciones/relaciones_china_alatina.pdf

Gómez, D. A. (2013). China y su relación con América Latina. Una aproximación desde el poder blando. En Martínez, J. I (coord.), América Latina y el CaribeChina. Relaciones políticas e internacionales (pp. 47-64). Ciudad de México: Unión de Universidades de América Latina y el Caribe.

Hernández, C. (2013). Las relaciones entre China y Latinoamérica en la década de los 2010. En Martínez, J. I (coord.), América Latina y el Caribe-China. Relaciones politicas e internacionales (pp. 121-137). Ciudad de México: Unión de Universidades de América Latina y el Caribe.

Ibáñez, L. M., \& Dávila, D. C. (2010). Colombia y China: treinta años de amistad y cooperación. Bogotá: Ministerio de Relaciones Exteriores.

Instituto Confucio Utadeo. (2013). Instituto Confucio Utadeo. Bogotá: Utadeo. Mesa, B., \& González, P. (2016). (2016). Paradiplomacia: una herramienta de poder blando de China. Papel Político, 21(2), 537-563. Recuperado de http:// revistas.javeriana.edu.co/index.php/papelpol/article/viewFile/19072/14947. https://doi.org/10.11144/Javeriana.papo21-2.phpb 
Nye, J. S. (2003). La paradoja del poder norteamericano. Madrid: Santillana.

Oficina General del Instituto Confucio [Hanban]. (2016). Institutos Confucio. Recuperado de http://www.hanban.edu.cn/confuciousinstitutes/ node_10961.htm

Ramírez, F. (2016). Influencia del Confucianismo en la política exterior de la República Popular de China (tesis de pregrado). Universidad de Bogotá Jorge Tadeo Lozano, Bogotá.

Rodríguez, I., \& Leiva, D. (2013). El soft power en la política exterior de China: consecuencias para América Latina. Polis. Revista Latinoamericana, (35). Recuperado de http://polis.revues.org/9179. https://doi.org/10.4067/S0718-65682013000200022

Xu, S. (2013). China: seguro socio estratégico de América Latina y el Caribe. En Martínez, J. I (coord.), América Latina y el Caribe-China. Relaciones políticas e internacionales (pp. 139-154). Ciudad de México: Unión de Universidades de América Latina y el Caribe. 